\title{
Mitophagy: a mechanism for plant growth and survival
}

Martyna Broda ${ }^{1}$, A. Harvey Millar ${ }^{1}$ and Olivier Van Aken $^{2}$

${ }^{1}$ ARC Centre of Excellence in Plant Energy Biology, University of Western Australia, Crawley, Western Australia, Australia.

${ }^{2}$ Department of Biology, Lund University, Sölvegatan 35, 22362 Lund, Sweden

Corresponding author:

olivier.van aken@biol.lu.se (O. Van Aken)

http://www.biology.lu.se/olivier-van-aken

Keywords: mitochondria, autophagy, senescence, reactive oxygen species, plant hormones, cell death 


\begin{abstract}
Mitophagy is a conserved cellular process important for the autophagic removal of damaged mitochondria to maintain a healthy mitochondrial population. Mitophagy appears to occur also in plants and has roles in development, stress response, senescence and programmed cell death. However, many of the genes that control mitophagy in yeast and animal cells are absent in plants, and no plant proteins marking defunct mitochondria for autophagic degradation are yet known. New insights implicate general autophagy-related proteins in mitophagy, affecting the senescence of plant tissues. Mitophagy control and its importance for energy metabolism, survival, signalling and cell death in plants are discussed. Furthermore, we suggest mitochondrial membrane proteins containing ATG8-interacting motifs, which might serve as mitophagy receptor proteins in plant mitochondria.
\end{abstract}




\section{Glossary}

ATG: AuTophaGy-related proteins

Autophagosome: a double layer membrane structure involved in macroautophagy, the intracellular degradation system for cytoplasmic contents.

Chlorophagy: the selective degradation of chloroplasts by autophagy.

Isolation membrane: synonym for phagophore.

Mitophagy: the selective degradation of mitochondria by autophagy.

Non-selective autophagy: bulk autophagy to degrade various cellular components without specificity.

PAS: pre-autophagosomal structure, the putative site for autophagosome formation

PCD: Programmed cell death

Phagophore: a double membrane that encloses and isolates the cytoplasmic components during macroautophagy, also called isolation membrane

Retrograde signaling: cellular signaling from mitochondria or chloroplasts to the cellular nucleus, triggering changes in nuclear gene expression.

Selective autophagy: the selective autophagy of specific organelles or cellular structures 


\section{Mitophagy as a type of autophagy}

Autophagy is the process of controlled recycling of cellular contents and organelles to promote cell survival or redistribute nutrients. In normal cellular conditions autophagy may recycle components that accumulate for example oxidative damage [1], but its rate can drastically be increased under a variety of stress conditions, senescence and cell death [2-4]. During autophagy, portions of the cytoplasm are captured in vesicles (autophagosomes; see glossary) and degraded in lysosomes (animals) or the vacuole (Saccharomyces cerevisiae and plants) [5]. Many cellular organelles have been described to undergo autophagy, including the endoplasmic reticulum (ER), the nucleus, mitochondria (mitophagy) and chloroplasts (chlorophagy) [6-8]. This review will particularly focus on mitophagy in plants. We define this as the process of mitochondrial degradation through autophagy-related processes, not the role of mitochondria during general autophagy. Mitochondria are crucial for energy metabolism, biosynthesis, regulation of cell death and are also involved in stress response and intracellular signalling [9-11]. A key component of mitochondrial function is the electron transport chain (ETC), which despite its beneficial roles is also a major source of reactive oxygen species (ROS) production that can lead to oxidative damage [12]. Moreover, dysfunctional mitochondria consume cytosolic ATP, resulting in energy losses [13]. Therefore, the controlled removal of dysfunctional or superfluous mitochondria by mitophagy is important for maintaining a healthy mitochondrial population. In C. elegans mitophagy is involved in coordination of mitochondrial biogenesis, recycling of Fe-S clusters during Fe starvation and has implications for longevity and ageing [14, 15].

Moderate rates of autophagy thus promote cell survival, while excessive autophagy can lead to cell death in most organisms, including in plants [16-18]. In plants, mitochondria and chloroplasts are both subject to autophagy, and several AuTophaGy (ATG) genes have been implicated in these processes $[7,8,19]$. However, the molecular mechanisms of mitophagy in 
plants and how the process or the key components are differentiated from chlorophagy need to be investigated further.

\section{What is the evidence for mitophagy in plants?}

Different types of autophagy have been described in plants based on ultrastructural observations [20]. During microautophagy the vacuolar membrane engulfs a portion of the cytoplasm and buds off, forming a membrane-bound vesicle inside the vacuole. By contrast, macroautophagy takes place outside the vacuole by formation of double-membrane autophagosomes. Another autophagy-related phenomenon in plants has been termed megaautophagy or mega-autolysis, which refers to the extensive breakdown observed at the end of developmental programmed cell death (PCD), but it is debated whether this is a true form of autophagy [20, 21]. Plant specific types of autophagy involving the chloroplasts are also reported to occur. Small vesicles called RuBisCO-containing bodies (RCBs) move from the chloroplasts to the vacuole, before the whole organelle moves, in order to quickly recycle RuBisCO (a major nitrogen sink in plants), in a process requiring AuTophaGy-related (ATG) protein ATG5 and involving ATG8 [22]. Interestingly, chloroplasts have also been reported to perform autophagic tasks by engulfing portions of the cytoplasm and degrading this content inside the chloroplast $[23,24]$.

The occurrence of mitophagy in yeast and animals is well-established, however this field of study in plants is still in its early stage. An early study reported mitochondria being enclosed in a double-membrane structure similar to ER during autophagy in mung bean (Vigna radiata) [25]. These autophagosome-like structures containing mitochondria were observed to fuse with lytic vacuoles. Numerous autophagosomes enclosing mitochondria have been described after one day of tracheary element differentiation in xylem [26]. Wertman and colleagues reported that aggregates of mitochondria can be observed inside the vacuole during later stages of 
developmental PCD in the lace plant (Aponogeton fenestralis) [27]. A study characterising accelerated cell death 5 mutants reported mitochondrial ROS formation and the presence of mitochondria in autophagosomes [28]. Also Minibayeva and colleagues demonstrated both intact and partly degraded mitochondria in the vacuole after methyl viologen treatment of wheat roots [2]. However, another study criticized this claim and suggests the authors were not observing mitophagy, but rather mitochondria in cytoplasmic strands [20]. The observation of mitochondria in vacuoles and lytic vesicles should nevertheless be taken cautiously as evidence for mitophagy, as direct analysis of organelle degradation kinetics is required to conclude that autophagy is selective towards a certain type of organelle.

Recently, it was reported that during senescence mitochondrial proteins and mitochondrial vesicles were degraded by autophagy (mitophagy) in arabidopsis (Arabidopsis thaliana) [19]. Studies of mitochondrial protein degradation rate have been performed in arabidopsis cell cultures [29] and arabidopsis plants [30], and both reveal a basal rate of mitochondrial protein removal from plants cells of approximately 5-10\% per day, analogous to the rates of mitochondrial turnover in some yeast and mammalian cells [31, 32]. Loss of the Lon1 mitochondrial matrix protease in plants led to an increase in mitochondrial turnover for a large number of respiratory-related proteins that could indicate induction of mitophagy [33]. Chloroplasts have even been observed to invaginate mitochondria to degrade them internally, as an alternative means of mitophagy [34], however to the date no independent reports of this phenomenon exist. In summary, it appears a number of studies in plants have observed processes analogous to mitophagy and provide evidence that this is an actively controlled process (Box 1).

\section{The mechanism of mitophagy in plant and non-plant systems}

Both non-selective and selective autophagy (such as mitophagy) can be divided into phases: 1) initiation, 2) recognition of cargo, 3) nucleation and phagophore (the double membrane that 
encloses cytoplasmic components during macroautophagy) formation, 4) autophagosome maturation, 5) delivery of cargo and finally 6) degradation in the vacuole (in yeast and plants) or in lysosomes (in mammals). These processes are tightly controlled by signalling pathways, which involve ATG proteins, membrane structures and marker proteins, as well as regulation of degradation systems (i.e. vacuoles or lysosomes). Furthermore, post-translational modifications play a role in recruiting and targeting the autophagy complexes (see Box 1 and Figure 1).

\section{Non-plant systems}

The initiation of a mitophagosome (an autophagosome engulfing a mitochondrion) requires targeting of mitochondria for degradation and the formation of an initial isolation membrane. In yeast, mitophagy involves the mitochondrial proteins ATG32 or ATG33 [35, 36]. ATG32 is located in the mitochondrial outer membrane and acts as a receptor, recruiting other ATG proteins which are essential for the initial isolation membrane formation. ATG32 recruits ATG8 and ATG11 is phosphorylated by CK2 (casein kinase 2) to stabilise the ATG32-ATG11 interaction [37, 38]. Together with the core ATG proteins, the ATG32-ATG11 complex generates the isolation membrane to engulf a mitochondrion [6]. In yeast, ATG11 is part of the ATG1/13 complex along with ATG101, ATG17 and the yeast specific proteins ATG29 and ATG32 [39, 40].

In mammals, at least two distinct mitophagy pathways exist. One pathway that occurs in mammalian cells involves hypoxia- or uncoupling-induced phosphorylation of the outer mitochondrial membrane protein FUNDC1 by the ATG1 homologue ULK1, resulting in mitophagy [41]. A second pathway in mammalian systems is dependent on the mitochondrial transmembrane potential and is affected in Parkinson's disease. In healthy mitochondria, the kinase PINK1 is partially imported through the TOM complex and across the mitochondrial inner membrane (IMM) in a $\Delta \psi_{\mathrm{m}}$-dependent manner. There, PINK1 is degraded by pre-senilin 
associated rhomboid-like protease PARL [42]. In damaged mitochondria where $\Delta \psi_{\mathrm{m}}$ is reduced, PINK1 remains active at the outer mitochondrial membrane (OMM) where it phosphorylates the E3 ubiquitin ligase Parkin. Parkin can then ubiquitinylate multiple proteins on the mitochondrial surface including voltage-dependent anion channel VDAC1 [43], which eventually leads to core ATG protein recruitment and mitophagy. Although PINK1/parkin are not present in plant genomes (Table 1), there might be functional analogies with plant FRIENDLY in Arabidopsis thaliana [44]. FRIENDLY1 is an ortholog of Clueless in Drosophila melanogaster, and deletion of either protein causes a severe clustering of mitochondria within the cytoplasm. As Clueless is required for mitophagy in concert with Parkin [45, 46], this topic needs to be further investigated in plants.

The origin of the autophagosomal membrane is still under investigation but significant evidence exists that autophagosomes can arise from plasma membrane, Golgi, endosomes, as well as from ER and/or mitochondrial membranes [47-50]. In mammalian systems, ERmitochondria contact sites may be of key importance for autophagosome formation during starvation, involving recruitment of ATG14 and ATG5 proteins. Mitophagy is observed at ERmitochondria contact sites via mitochondria-associated membranes (MAMs) that derive from the ER [51, 52]. Disruption of these MAMs can inhibit autophagosome formation. The ER may thus provide the platform for autophagosome formation, with the mitochondria contributing other components required for the process [52]. These ER-mitochondria contact sites are maintained, for example by the ER-mitochondria encounter structure (ERMES) complex in yeast [53]. It was shown that under starvation conditions mitochondrial and autophagosomal membranes becomes continuous [48], although the reason behind this starvation specificity remains unclear. It is also known that mitochondria and ER share contact sites that are required for mitophagy in yeasts [54]. 
Although some of the mechanisms described above may conceptually be similar in plants, there is very little conservation of mitophagy regulators between yeast/animals and plants. Most of the core ATG genes are conserved in plants $[16,55]$, but the specific players in mitophagy are largely absent in the Arabidopsis thaliana genome (Table 1), such as yeast ATG32. Also key mammalian regulators such as DCT-1 (BNIP3, related to BCL-2) that act as mitophagy receptors in mammals, are not present in plant genomes. Recently, a homolog of yeast ATG11 (and animal FIP200) has been found in Arabidopsis thaliana and has been proven to be involved in mitophagy under nitrogen-starvation conditions $[19,56]$. The sequence homology between yeast and plant ATG11 is however low (20\% identity), with AtATG11 containing traces of both ATG11 and ATG17 domains (see Table 1). In arabidopsis, ATG11 interacts directly with ATG8 (homologous to mammalian LC3), ATG13 and ATG10 [19]. The interaction between ATG11 and ATG1 is indirect and led by ATG13 (Fig. 1). ATG11 is thought to help link the ATG1/13 complex and to promote the delivery of vesicles to the vacuole, however ATG11 is not completely essential for the assembly of the autophagic bodies [19]. ATG11 is also thought to be involved in the dynamic turnover of the ATG1/ATG13 kinase complex during nutrient starvation [57][19].

Also ATG7 is important during senescence-induced mitophagy [19]. ATG7 is an E1-like enzyme which mediates the conjugation of ATG8 with phosphatidylethanolamine (PE) and of ATG12 with ATG5, resulting in the formation of ATG8-PE and ATG5-ATG12 complexes [58]. The ATG5-ATG12-ATG16L complex (an E3-like enzyme) is responsible for lipidation of ATG8 by PE $[39,59]$. The atg8-PE adduct decorates the mature autophagosomal membrane (Fig. 1), making it a good marker for the observation of autophagosome formation also in plants $[39,58,60]$.

With regards to autophagosome membrane formation, ATG5 and ATG8 are recruited during phagophore formation in close association with the endoplasmic reticulum (ER) [61]. Recently, the autophagy protein ATG9 has been shown to be important in the regulation of 
autophagosome membrane progression from the ER [62]. The loss of function atg9 mutants in arabidopsis displayed unusual tubular structures extending from the ER upon induction of autophagy. This phenomenon has not been observed in other autophagy mutants. Loss of ATG9 in arabidopsis does not affect ATG8 conjugation onto the autophagosomal membrane, indicating a role for ATG9 downstream of initial ATG8 recruitment. The role of ATG9 in this process currently appears to be unique to arabidopsis (and perhaps other plant systems), and stands opposite to other organisms where loss of function atg9 mutants fail in autophagosome formation [63].

\section{Mitochondrial membrane autophagy receptors in plants}

Based on our current knowledge, it appears that specific proteins on the mitochondrial surface act as markers for degradation and recruit the autophagy machinery to a specific mitochondrion. In yeast, ATG32 is an OMM receptor involved in tagging mitochondria for autophagosomal degradation, however to date there are no proteins on the plant mitochondrial surface that have been experimentally confirmed to have a similar function. Recently, a bioinformatic tool was developed to predict proteins that may interact with ATG8 [64], a core protein of autophagy machinery. Based on the set of experimentally-determined OMM proteins in arabidopsis [65], a predicted ATG8-interacting protein set [62] for arabidopsis comprises 12 proteins including cytochrome b reductase, hexokinase 1, translocases of the OMM (TOM20s and TOM40) and voltage-dependent anion channel VDAC2 (Table 2). Mitochondrial protein import plays an important role in control of autophagy in animal systems, so perhaps a similar phenomenon occurs in plants explaining the presence of TOM20/40 in this list [42]. Furthermore, VDAC1 ubiquitination by Parkin is a crucial step in marking mitochondria for autophagy [43] in animals, and also hexokinase plays an autophagy-promoting role via the TOR pathway [66], potentially explaining these proteins being in the predicted ATG8interacting protein set. 
Prohibitin 2 was identified as an IMM receptor in animal systems, which interacts with LC3/ATG8 [67]. This interaction requires the rupture of the OMM first, which occurs during Parkin-mediated autophagy [67, 68]. Amongst arabidopsis IMM proteins (213 IMM proteins based on SUBA4 [69] and Uniprot [70]) another set of 36 predicted ATG8-interacting proteins [62] can be identified (Table 2). This list includes various ETC, ATP synthase and Translocon of IMM (TIM) proteins, FtsH4/11 proteases [71], mitochondrial calcium uniporters MCU1/6 [72], metabolite transporters, but not prohibitins. However, ATG8 interaction motifs [62] were not identified in animal PHB2 either, so a role for plant prohibitins in mitophagy should not be ruled out. As loss of prohibitins in plants results in a range of mitochondrial defects, mitochondrial swelling and retrograde signalling responses, it will be interesting to find if mitophagy is affected in prohibitin mutants and mitochondrial mutants in general [73-75].

\section{Role for rhomboid and other proteases}

Rhomboid proteases are a specific group of conserved sequence-specific intramembrane proteases [76]. Mitochondrially targeted members are involved in PCD and autophagy in animal systems. For instance, PARL prevents apoptosis by activation of Omi1 and by preserving cristae structure to prevent cytochrome c release [77]. It is also involved in suppressing mitophagy by cleaving PINK1 [76]. Plant genomes also encode rhomboid-like proteases (e.g. 15 putatively in arabidopsis), and some rhomboid proteases are also present in the mitochondrial and chloroplast membranes [78, 79]. Chloroplast rhomboids may be involved in maturation of Tic40 import component [80]. However, no drastic phenotypes have been observed in single or double mutants of AtRBL8 and AtRBL9, with some partial sterility defects in atrbl8 plants that may be attributed to decreased expression of jasmonic acid (JA) synthase allene oxide synthase [81]. Thus, no significant evidence exists that chloroplast or mitochondrial rhomboid proteases play an active role in plant autophagy or PCD. 
An arabidopsis mitochondrial protease (AtPARK13) with similarity to animal Park13/Omi/HtrA (a substrate of autophagy-activator PINK1) has been reported to have a role in thermotolerance [82]. The authors suggest it can directly cleave substrates, but no involvement in autophagy per se has been proven.

Yeast iAAA/FtsH-like proteases are involved in cleaving the C-terminal of mitophagy receptor ATG32, thereby stimulating autophagy potentially by improving the interaction of ATG32 with ATG11 [83]. In arabidopsis, loss of mitochondrial protease Ftsh4 caused severe leaf senescence, cell death, and increased autophagy levels [71]. ATG5 and ATG8 were required for autophagosome formation and the senescence phenotype of $f t s h 4$ mutants. Crossing of $f t s h 4$ with salicylic acid (SA) signalling-deficient mutants reversed the senescence and autophagy phenotypes, suggesting an important role of SA. Also a role for WRKY transcription factors was suggested.

Several other mitochondrial proteases are induced at a transcript level during conditions that are linked with autophagy induction, such as senescence (ClpB4, all three ClpX's, and ClpB2), dark treatment (Lon2) and nitrogen starvation (ClpP2 and metacaspase MC3) [84-87]. It will therefore be interesting to determine in the future if these proteases are involved in mitophagy induction or progression in plants.

\section{The role of mitophagy in signalling}

The communication from mitochondria to nucleus has been studied intensively in plants and some components in this retrograde signalling have been identified [10]. Understanding how this communication is coordinated might be a key to understand the outcomes of different cellular responses and their link to autophagic processes in plants. Generic ROS signalling in cells is likely not specific enough to induce targeted nuclear transcriptional changes in response to specific organelle defects, rather, receptors of specific ROS signals might be needed [88]. One possibility in the case of mitochondria is the ROS-dependent induction of the unfolded 
protein response $\left(\mathrm{UPR}_{\mathrm{mt}}\right)$, which has been studied in non-plant systems [89]. The precise mechanisms of UPRmt in plants are only beginning to be understood [90], but it seems plausible that mitophagy could be involved. For instance the mammalian mitochondrial deacetylase sirtuin SirT3 is a regulator of both UPRmt and mitophagy [91, 92]. It is thought that SirT3 helps to sort moderately stressed mitochondria from irreversibly damaged ones. From previous studies it is known that a similar unfolded protein response occurs in the endoplasmic reticulum (UPR $\mathrm{ER})$ and can activate formation of autophagosomes in plants [51, 93, 94]. ER and mitochondria interact through junctions on the ER membrane [54], and significant evidence exists that autophagosomal membranes can be derived from both ER [47, 62] and mitochondria (at least in non-plant systems, as discussed above) [47]. Mitophagy may also take part in retrograde signal suppression, for instance by removing damaged organelles that may be sending out stress signals. At least in animal systems, suppression of mitophagy results in retrograde signalling that regulates mitochondrial biogenesis [15]. The role of mitochondria in oxidative stress-induced autophagy in plants has been previously reviewed [2], further highlighting specific areas of research that are needed to understand the impact of mitophagy on plant mitochondrial function and signalling.

\section{Role of mitophagy during senescence}

Most arabidopsis mutants lacking autophagy-related genes have no clear early developmental phenotypes, except atg6 mutants that have pollen germination defects [16]. However, lack of autophagy often results in accelerated senescence in arabidopsis [8, 19, 95, 96]. Furthermore, dark induced senescence causes chlorophagy, which requires ATG4, although no abnormal whole-plant senescent phenotypes were observed in atg4a4b-1 arabidopsis mutants [96]. Chlorophagy is of major importance for nitrogen recycling as $80 \%$ of cellular nitrogen is held in the chloroplasts [96], as well as during recovery from UV-induced damage [97]. Chloroplasts are degraded much earlier than mitochondria during senescence. Mitochondria 
are possibly retained longer for recycling nitrogen via $\mathrm{NH}_{4}{ }^{+}$by glutamate dehydrogenase [95]. As sugars are depleted rapidly in a senescing leaf and amino acids need to be recycled, glutamate and branched chain amino acids such as lysine can feed electrons into the mitochondrial electron transport chain, keeping metabolism and nutrient recycling going [95, 98, 99]. A recent study showed that during dark-induced senescence concentrations of most amino acids increased, but this was less pronounced in atg mutants [100]. On the other hand, TCA cycle intermediates such as citrate were more abundant and dark respiration rate was higher in atg mutants than in WT plants. The atg mutant plants responded to dark-induced senescence by increasing transcripts of alternative mitochondrial respiration pathway enzymes ETF/ETFQO. This suggests a metabolic reorientation when autophagy is disrupted, and that the lack of protein degradation in atg mutants slowed the generation of amino acids used as alternative substrates for respiration [100]

After one day of dark-induced senescence a significant increase in ROS production by mitochondria and peroxisomes has been observed that lasted throughout senescence [101], possibly reflecting the heightened activity of these organelles during senescence. In contrast, chloroplast ROS levels dropped after 1 day and gradually returned to basal levels over the course of senescence [102]. Based on this, it could be speculated that mitochondria (and perhaps peroxisomes) are the main players that allow complete recycling of cell content and potentially lead to cell death at the end of plant senescence. In agreement, plant mitochondria keep moving actively around the cell [102] and maintain their function [103] until the last stages of senescence when chlorophyll is already largely degraded. This implies that cell survival through mitochondrial metabolic function until the last moments of senescence is crucial to maximise nutrient remobilisation [103] (Figure 2). When the time for cell death in plants has arrived, it is unclear how the PCD threshold is reached, and if mitochondria and their autophagic removal play an active role (Box 2). It is possible that mitochondrial degradation is the final step in completion of senescence, or alternatively that they simply run out of substrates 
to maintain cellular viability. One explanation for the observed accelerated senescence phenotype in plant mitophagy mutants atg11 [19] may be that high activity and observed ROS production of mitochondria in senescing leaves requires adequate mitochondrial quality control and the removal of damaged organelles. When these damaged organelles accumulate they lose optimal functionality resulting in premature senescence. In agreement, ATG11 transcripts are gradually upregulated during leaf senescence in arabidopsis, peaking during the final stages (Box 1 Figure I).

Autophagy and potentially mitophagy may also play a role in ageing and lifespan extension in plants. Low light conditions can induce lifespan extension via caloric restriction in arabidopsis, and autophagy supports this extended lifespan by efficient recycling of contents, [104]. Also in animals, it is thought that a decline in mitophagy and thus mitochondrial quality control may contribute to aging $[15,105]$.

\section{Conclusions}

Current evidence suggests that mitophagy occurs in plants both during normal development and under conditions such as prolonged darkness and oxidative stress (Figure 2). At present, only limited experimental information is available on how mitophagy contributes to suppressing premature senescence in plants, and whether mitophagy and plant PCD are linked $[5,18,19]$. An emerging model suggests that mitochondria are needed to allow efficient recycling and remobilisation of nutrients for instance in senescent leaves (Figure 2). This might put significant pressure on mitochondrial energy systems, thus requiring efficient removal of damaged and 'worn-out' organelles. If this turnover mediated by mitophagy is inhibited, the plants may senesce without complete remobilisation of nutrients. Thus, removing damaged, potentially ROS-overproducing energy organelles may promote cell survival, and may contribute to the natural turnover of ageing mitochondria. During stress, it appears that ROS 
such as superoxide may be a signal that triggers autophagy to remove organelles that are engaged in excessive ROS production [2].

Mechanistically, we understand only a little about how mitophagy in plants is executed. Many of the core ATG protein components appear to be conserved in plants, but we have virtually no evidence of how individual plant mitochondria are marked for removal by autophagy. We hope that the list presented in Table 2 will be a useful resource for guiding such studies in the future. There is a need for further development of mitophagy tools in plants such as reporter lines and antibodies against proteins that are specifically degraded in plant mitochondria by autophagy [106, 107]. We also have very little understanding of how plant mitophagy could be involved in regulating cellular processes outside of senescence, such as general tissue maintenance, gamete development, developmental processes that involve cell removal, and whether mitophagy plays a role in stimulating or quenching stress-related signalling pathways in plants. 


\section{Acknowledgements}

AHM and MB are funded by support from the ARC Centre of Excellence in Plant Energy Biology (CE140100008). O.V.A and A.H.M. were supported by Australian Research Council Discovery grant (DP160103573). OVA was supported by the Swedish Research Council (VR 2017-03854), Crafoord Foundation (20170862) and Carl Trygger Foundation (CTS17-487). 


\section{References}

1. Xiong, Y. et al. (2007) Degradation of oxidized proteins by autophagy during oxidative stress in Arabidopsis. Plant Physiol 143 (1), 291-9.

2. Minibayeva, F. et al. (2012) Oxidative stress-induced autophagy in plants: the role of mitochondria. Plant Physiol Biochem 59, 11-9.

3. Marino, G. et al. (2014) Self-consumption: the interplay of autophagy and apoptosis. Nat Rev Mol Cell Biol 15 (2), 81-94.

4. Liu, Y. et al. (2009) Autophagy is required for tolerance of drought and salt stress in plants. Autophagy 5 (7), 954-63.

5. Minina, E.A. et al. (2014) Autophagy as initiator or executioner of cell death. Trends Plant Sci 19 (11), 692-697.

6. Okamoto, K. (2014) Organellophagy: eliminating cellular building blocks via selective autophagy. J Cell Biol 205 (4), 435-45.

7. Spitzer, C. et al. (2015) The Endosomal Protein CHARGED MULTIVESICULAR BODY PROTEIN1 Regulates the Autophagic Turnover of Plastids in Arabidopsis. Plant Cell 27 (2), 391-402.

8. Sakuraba, Y. et al. (2014) Delayed degradation of chlorophylls and photosynthetic proteins in Arabidopsis autophagy mutants during stress-induced leaf yellowing. J Exp Bot 65 (14), 3915-25.

9. Van Aken, O. and Van Breusegem, F. (2015) Licensed to kill: mitochondria, chloroplasts, and cell death. Trends Plant Sci in press.

10. Ng, S. et al. (2014) Anterograde and retrograde regulation of nuclear genes encoding mitochondrial proteins during growth, development, and stress. Mol Plant 7 (7), 1075-93.

11. Millar, A.H. et al. (2008) Mitochondrial biogenesis and function in Arabidopsis. Arabidopsis Book 6, e0111.

12. Huang, S. et al. (2016) The Roles of Mitochondrial Reactive Oxygen Species in Cellular Signaling and Stress Response in Plants. Plant Physiol 171 (3), 1551-9.

13. Gomes, L.C. and Scorrano, L. (2013) Mitochondrial morphology in mitophagy and macroautophagy. Biochim Biophys Acta 1833 (1), 205-12.

14. Schiavi, A. et al. (2015) Iron-Starvation-Induced Mitophagy Mediates Lifespan Extension upon Mitochondrial Stress in C. elegans. Curr Biol 25 (14), 1810-22.

15. Palikaras, K. et al. (2015) Coordination of mitophagy and mitochondrial biogenesis during ageing in C. elegans. Nature 521 (7553), 525-8.

16. Reumann, S. et al. (2010) From signal transduction to autophagy of plant cell organelles: lessons from yeast and mammals and plant-specific features. Protoplasma 247 (3-4), 233-56.

17. Minina, E.A. et al. (2013) Autophagy and metacaspase determine the mode of cell death in plants. J Cell Biol 203 (6), 917-27.

18. Yoshimoto, K. et al. (2009) Autophagy negatively regulates cell death by controlling NPR1dependent salicylic acid signaling during senescence and the innate immune response in Arabidopsis. Plant Cell 21 (9), 2914-27.

19. Li, F. et al. (2014) AUTOPHAGY-RELATED11 plays a critical role in general autophagy- and senescence-induced mitophagy in Arabidopsis. Plant Cell 26 (2), 788-807.

20. van Doorn, W.G. and Papini, A. (2013) Ultrastructure of autophagy in plant cells: a review. Autophagy 9 (12), 1922-36.

21. van Doorn, W.G. (2011) Classes of programmed cell death in plants, compared to those in animals. J Exp Bot 62 (14), 4749-61.

22. Ishida, H. et al. (2008) Mobilization of rubisco and stroma-localized fluorescent proteins of chloroplasts to the vacuole by an ATG gene-dependent autophagic process. Plant Physiol 148 (1), 142-55.

23. van Doorn, W.G. et al. (2011) Do plastids in Dendrobium cv. Lucky Duan petals function similar to autophagosomes and autolysosomes? Autophagy 7 (6), 584-97.

24. Filonova, L.H. et al. (2000) Two waves of programmed cell death occur during formation and development of somatic embryos in the gymnosperm, Norway spruce. J Cell Sci 113 Pt 24, 4399-411. 
25. Toyooka, K. et al. (2001) Cotyledon cells of Vigna mungo seedlings use at least two distinct autophagic machineries for degradation of starch granules and cellular components. J Cell Biol 154 (5), 973-82.

26. Kwon, S.I. et al. (2010) The Rab GTPase RabG3b functions in autophagy and contributes to tracheary element differentiation in Arabidopsis. Plant J 64 (1), 151-64.

27. Wertman, J. et al. (2012) The pathway of cell dismantling during programmed cell death in lace plant (Aponogeton madagascariensis) leaves. BMC Plant Biol 12, 115.

28. Bi, F.C. et al. (2014) Loss of ceramide kinase in Arabidopsis impairs defenses and promotes ceramide accumulation and mitochondrial $\mathrm{H} 2 \mathrm{O} 2$ bursts. Plant Cell 26 (8), 3449-67.

29. Nelson, C.J. et al. (2013) Degradation rate of mitochondrial proteins in Arabidopsis thaliana cells. J Proteome Res 12 (7), 3449-59.

30. Li, L. et al. (2017) Protein Degradation Rate in Arabidopsis thaliana Leaf Growth and Development. Plant Cell.

31. Kim, T.Y. et al. (2012) Metabolic labeling reveals proteome dynamics of mouse mitochondria. Mol Cell Proteomics 11 (12), 1586-94.

32. Pratt, J.M. et al. (2002) Dynamics of protein turnover, a missing dimension in proteomics. Mol Cell Proteomics 1 (8), 579-91.

33. Li, L. et al. (2017) Changes in specific protein degradation rates in Arabidopsis thaliana reveal multiple roles of Lon1 in mitochondrial protein homeostasis. Plant J 89 (3), 458-471.

34. Ragetli, H.W. et al. (1970) Degeneration of leaf cells resulting from starvation after excision. I. Electron microscopic observations. Can. J. Bot 48, 1913-1922.

35. Kanki, T. et al. (2009) A genomic screen for yeast mutants defective in selective mitochondria autophagy. Mol Biol Cell 20 (22), 4730-8.

36. Okamoto, K. et al. (2009) Mitochondria-anchored receptor Atg32 mediates degradation of mitochondria via selective autophagy. Dev Cell 17 (1), 87-97.

37. Aoki, Y. et al. (2011) Phosphorylation of Serine 114 on Atg32 mediates mitophagy. Mol Biol Cell 22 (17), 3206-17.

38. Kanki, T. et al. (2013) Casein kinase 2 is essential for mitophagy. EMBO Rep 14 (9), 788-94.

39. Ryabovol, V.V. and Minibayeva, F.V. (2016) Molecular Mechanisms of Autophagy in Plants: Role of ATG8 Proteins in Formation and Functioning of Autophagosomes. Biochemistry (Mosc) 81 (4), 348-63.

40. Thompson, A.R. and Vierstra, R.D. (2005) Autophagic recycling: lessons from yeast help define the process in plants. Curr Opin Plant Biol 8 (2), 165-73.

41. Wu, W. et al. (2014) ULK1 translocates to mitochondria and phosphorylates FUNDC1 to regulate mitophagy. ЕMBO Rep 15 (5), 566-75.

42. Jin, S.M. et al. (2010) Mitochondrial membrane potential regulates PINK1 import and proteolytic destabilization by PARL. J Cell Biol 191 (5), 933-42.

43. Geisler, S. et al. (2010) PINK1/Parkin-mediated mitophagy is dependent on VDAC1 and p62/SQSTM1. Nat Cell Biol 12 (2), 119-31.

44. Logan, D.C. et al. (2003) The genetic control of plant mitochondrial morphology and dynamics. Plant J 36 (4), 500-9.

45. Cox, R.T. and Spradling, A.C. (2009) Clueless, a conserved Drosophila gene required for mitochondrial subcellular localization, interacts genetically with parkin. Dis Model Mech 2 (9-10), 490-9.

46. Wang, Z.H. et al. (2016) Drosophila clueless is involved in Parkin-dependent mitophagy by promoting VCP-mediated Marf degradation. Hum Mol Genet 25 (10), 1946-1964.

47. Chan, S.N. and Tang, B.L. (2013) Location and membrane sources for autophagosome formation from ER-mitochondria contact sites to Golgi-endosome-derived carriers. Mol Membr Biol 30 (8), 394402.

48. Hailey, D.W. et al. (2010) Mitochondria supply membranes for autophagosome biogenesis during starvation. Cell $141(4), 656-67$. 
49. Lamb, C.A. et al. (2013) The autophagosome: origins unknown, biogenesis complex. Nat Rev Mol Cell Biol 14 (12), 759-74.

50. Mueller, S.J. and Reski, R. (2015) Mitochondrial Dynamics and the ER: The Plant Perspective. Front Cell Dev Biol 3, 78.

51. Senft, D. and Ronai, Z.A. (2015) UPR, autophagy, and mitochondria crosstalk underlies the ER stress response. Trends Biochem Sci 40 (3), 141-8.

52. Hamasaki, M. et al. (2013) Autophagosomes form at ER-mitochondria contact sites. Nature 495 (7441), 389-93.

53. Kornmann, B. et al. (2009) An ER-mitochondria tethering complex revealed by a synthetic biology screen. Science 325 (5939), 477-81.

54. Bockler, S. and Westermann, B. (2014) Mitochondrial ER contacts are crucial for mitophagy in yeast. Dev Cell 28 (4), 450-8.

55. Meijer, W.H. et al. (2007) ATG genes involved in non-selective autophagy are conserved from yeast to man, but the selective Cvt and pexophagy pathways also require organism-specific genes. Autophagy 3 (2), 106-16.

56. Li, F. and Vierstra, R.D. (2014) Arabidopsis ATG11, a scaffold that links the ATG1-ATG13 kinase complex to general autophagy and selective mitophagy. Autophagy 10 (8), 1466-7.

57. Suttangkakul, A. et al. (2011) The ATG1/ATG13 protein kinase complex is both a regulator and a target of autophagic recycling in Arabidopsis. Plant Cell 23 (10), 3761-79.

58. Doelling, J.H. et al. (2002) The APG8/12-activating enzyme APG7 is required for proper nutrient recycling and senescence in Arabidopsis thaliana. J Biol Chem 277 (36), 33105-14.

59. Fujita, N. et al. (2008) The Atg16L complex specifies the site of LC3 lipidation for membrane biogenesis in autophagy. Mol Biol Cell 19 (5), 2092-100.

60. Liu, Y. and Bassham, D.C. (2012) Autophagy: pathways for self-eating in plant cells. Annu Rev Plant Biol 63, 215-37.

61. Le Bars, R. et al. (2014) ATG5 defines a phagophore domain connected to the endoplasmic reticulum during autophagosome formation in plants. Nat Commun 5, 4121.

62. Zhuang, X. et al. (2017) ATG9 regulates autophagosome progression from the endoplasmic reticulum in Arabidopsis. Proc Natl Acad Sci U S A 114 (3), E426-E435.

63. Yamamoto, H. et al. (2012) Atg9 vesicles are an important membrane source during early steps of autophagosome formation. J Cell Biol 198 (2), 219-33.

64. Xie, Q. et al. (2016) hfAIM: A reliable bioinformatics approach for in silico genome-wide identification of autophagy-associated Atg8-interacting motifs in various organisms. Autophagy 12 (5), 876-87.

65. Duncan, O. et al. (2011) Multiple lines of evidence localize signaling, morphology, and lipid biosynthesis machinery to the mitochondrial outer membrane of Arabidopsis. Plant Physiol 157 (3), 1093-113.

66. Roberts, D.J. et al. (2014) Hexokinase-II positively regulates glucose starvation-induced autophagy through TORC1 inhibition. Mol Cell 53 (4), 521-33.

67. Wei, Y. et al. (2017) Prohibitin 2 Is an Inner Mitochondrial Membrane Mitophagy Receptor. Cell 168 (1-2), 224-238 e10.

68. Chan, N.C. et al. (2011) Broad activation of the ubiquitin-proteasome system by Parkin is critical for mitophagy. Hum Mol Genet 20 (9), 1726-37.

69. Hooper, C.M. et al. (2017) SUBA4: the interactive data analysis centre for Arabidopsis subcellular protein locations. Nucleic Acids Res 45 (D1), D1064-D1074.

70. Apweiler, R. et al. (2004) UniProt: the Universal Protein knowledgebase. Nucleic Acids Res 32 (Database issue), D115-9.

71. Zhang, S. et al. (2017) The Arabidopsis Mitochondrial Protease FtSH4 Is Involved in Leaf Senescence via Regulation of WRKY-Dependent Salicylic Acid Accumulation and Signaling. Plant Physiol 173 (4), 2294-2307.

72. Teardo, E. et al. (2017) Physiological Characterization of a Plant Mitochondrial Calcium Uniporter in Vitro and in Vivo. Plant Physiol 173 (2), 1355-1370. 
73. Van Aken, O. et al. (2016) Retrograde signalling caused by heritable mitochondrial dysfunction is partially mediated by ANAC017 and improves plant performance. Plant J 88 (4), 542-558.

74. Van Aken, O. et al. (2007) Mitochondrial type-I prohibitins of Arabidopsis thaliana are required for supporting proficient meristem development. Plant J 52 (5), 850-64.

75. Piechota, J. et al. (2015) Unraveling the functions of type II-prohibitins in Arabidopsis mitochondria. Plant Mol Biol 88 (3), 249-67.

76. Chan, E.Y. and McQuibban, G.A. (2013) The mitochondrial rhomboid protease: its rise from obscurity to the pinnacle of disease-relevant genes. Biochim Biophys Acta 1828 (12), 2916-25.

77. Chao, J.R. et al. (2008) Hax1-mediated processing of HtrA2 by Parl allows survival of lymphocytes and neurons. Nature 452 (7183), 98-102.

78. Kmiec-Wisniewska, B. et al. (2008) Plant mitochondrial rhomboid, AtRBL12, has different substrate specificity from its yeast counterpart. Plant Mol Biol 68 (1-2), 159-71.

79. Knopf, R.R. et al. (2012) Rhomboid proteins in the chloroplast envelope affect the level of allene oxide synthase in Arabidopsis thaliana. Plant J 72 (4), 559-71.

80. Karakasis, K. et al. (2007) Uncovering a link between a plastid translocon component and rhomboid proteases using yeast mitochondria-based assays. Plant Cell Physiol 48 (4), 655-61.

81. Thompson, E.P. et al. (2012) An Arabidopsis rhomboid protease has roles in the chloroplast and in flower development. J Exp Bot 63 (10), 3559-70.

82. Basak, I. et al. (2014) Arabidopsis AtPARK13, which confers thermotolerance, targets misfolded proteins. J Biol Chem 289 (21), 14458-69.

83. Wang, K. et al. (2013) Proteolytic processing of Atg32 by the mitochondrial i-AAA protease Yme1 regulates mitophagy. Autophagy 9 (11), 1828-36.

84. Roberts, I.N. et al. (2012) Senescence-associated proteases in plants. Physiol Plant 145 (1), 130-9. 85. Liu, L. et al. (2008) Identification of early senescence-associated genes in rice flag leaves. Plant Mol Biol 67 (1-2), 37-55.

86. Lin, J.F. and Wu, S.H. (2004) Molecular events in senescing Arabidopsis leaves. Plant J 39 (4), 61228.

87. Sanmartin, M. et al. (2005) Caspases. Regulating death since the origin of life. Plant Physiol 137 (3), 841-7.

88. Moller, I.M. and Sweetlove, L.J. (2010) ROS signalling--specificity is required. Trends Plant Sci 15 (7), 370-4.

89. Haynes, C.M. and Ron, D. (2010) The mitochondrial UPR - protecting organelle protein homeostasis. J Cell Sci 123 (Pt 22), 3849-55.

90. Wang, X. and Auwerx, J. (2017) Systems Phytohormone Responses to Mitochondrial Proteotoxic Stress. Mol Cell 68 (3), 540-551 e5.

91. Liang, Q. et al. (2013) Bioenergetic and autophagic control by Sirt3 in response to nutrient deprivation in mouse embryonic fibroblasts. Biochem J 454 (2), 249-57.

92. Papa, L. and Germain, D. (2014) SirT3 regulates the mitochondrial unfolded protein response. Mol Cell Biol 34 (4), 699-710.

93. Liu, Y. et al. (2012) Degradation of the endoplasmic reticulum by autophagy during endoplasmic reticulum stress in Arabidopsis. Plant Cell 24 (11), 4635-51.

94. Pu, Y. and Bassham, D.C. (2013) Links between ER stress and autophagy in plants. Plant Signal Behav 8 (6), e24297.

95. Avila-Ospina, L. et al. (2014) Autophagy, plant senescence, and nutrient recycling. J Exp Bot 65 (14), 3799-811.

96. Wada, S. et al. (2009) Autophagy plays a role in chloroplast degradation during senescence in individually darkened leaves. Plant Physiol 149 (2), 885-93.

97. Izumi, M. et al. (2017) Entire Photodamaged Chloroplasts Are Transported to the Central Vacuole by Autophagy. Plant Cell 29 (2), 377-394.

98. Araujo, W.L. et al. (2010) Identification of the 2-hydroxyglutarate and isovaleryl-CoA dehydrogenases as alternative electron donors linking lysine catabolism to the electron transport chain of Arabidopsis mitochondria. Plant Cell 22 (5), 1549-63. 
99. Avin-Wittenberg, T. et al. (2015) Global analysis of the role of autophagy in cellular metabolism and energy homeostasis in Arabidopsis seedlings under carbon starvation. Plant Cell 27 (2), 306-22. 100. Barros, J.A. et al. (2017) Autophagy deficiency compromises alternative pathways of respiration following energy deprivation. Plant Physiol, in press.

101. Rosenwasser, S. et al. (2011) Organelles contribute differentially to reactive oxygen speciesrelated events during extended darkness. Plant Physiol 156 (1), 185-201.

102. Keech, O. (2011) The conserved mobility of mitochondria during leaf senescence reflects differential regulation of the cytoskeletal components in Arabidopsis thaliana. Plant Signal Behav 6 (1), 147-50.

103. Chrobok, D. et al. (2016) Dissecting the Metabolic Role of Mitochondria during Developmental Leaf Senescence. Plant Physiol 172 (4), 2132-2153.

104. Minina, E.A. et al. (2013) Autophagy mediates caloric restriction-induced lifespan extension in Arabidopsis. Aging Cell 12 (2), 327-9.

105. Diot, A. et al. (2016) Mitophagy plays a central role in mitochondrial ageing. Mamm Genome 27 (7-8), 381-95.

106. Bassham, D.C. (2015) Methods for analysis of autophagy in plants. Methods 75, 181-8.

107. Klionsky, D.J. et al. (2016) Guidelines for the use and interpretation of assays for monitoring autophagy (3rd edition). Autophagy 12 (1), 1-222.

108. Van Aken, O. and Pogson, B. (2017) Convergence of mitochondrial and chloroplastic ANAC017/PAP-dependent retrograde signalling pathways and suppression of programmed cell death. Cell Death Differ In press.

109. van der Graaff, E. et al. (2006) Transcription analysis of arabidopsis membrane transporters and hormone pathways during developmental and induced leaf senescence. Plant Physiol 141 (2), 77692.

110. Breeze, E. et al. (2011) High-resolution temporal profiling of transcripts during Arabidopsis leaf senescence reveals a distinct chronology of processes and regulation. Plant Cell 23 (3), 873-94.

111. Zhou, X.M. et al. (2015) A comprehensive, genome-wide analysis of autophagy-related genes identified in tobacco suggests a central role of autophagy in plant response to various environmental cues. DNA Res 22 (4), 245-57.

112. Deffieu, M. et al. (2013) Increased levels of reduced cytochrome b and mitophagy components are required to trigger nonspecific autophagy following induced mitochondrial dysfunction. J Cell Sci 126 (Pt 2), 415-26.

113. Popov, V.N. et al. (2003) Effect of Electron Transport Inhibitors on the Generation of Reactive Oxygen Species by Pea Mitochondria during Succinate Oxidation. Biochemistry (Moscow) 68 (7), 747-751.

114. Liu, L. et al. (2012) Mitochondrial outer-membrane protein FUNDC1 mediates hypoxia-induced mitophagy in mammalian cells. Nat Cell Biol 14 (2), 177-85.

115. Shiba-Fukushima, K. et al. (2012) PINK1-mediated phosphorylation of the Parkin ubiquitin-like domain primes mitochondrial translocation of Parkin and regulates mitophagy. Sci Rep 2, 1002. 116. Eiyama, A. and Okamoto, K. (2015) Protein N-terminal Acetylation by the NatA Complex Is Critical for Selective Mitochondrial Degradation. J Biol Chem 290 (41), 25034-44.

117. Kamada, Y. et al. (2000) Tor-mediated induction of autophagy via an Apg1 protein kinase complex. J Cell Biol 150 (6), 1507-13.

118. Chang, Y.Y. and Neufeld, T.P. (2009) An Atg1/Atg13 complex with multiple roles in TORmediated autophagy regulation. Mol Biol Cell 20 (7), 2004-14.

119. Puente, C. et al. (2016) Nutrient-regulated Phosphorylation of ATG13 Inhibits Starvationinduced Autophagy. J Biol Chem 291 (11), 6026-35.

120. Aubert, S. et al. (1996) Ultrastructural and biochemical characterization of autophagy in higher plant cells subjected to carbon deprivation: control by the supply of mitochondria with respiratory substrates. J Cell Biol 133 (6), 1251-63.

121. Kubli, D.A. and Gustafsson, A.B. (2012) Mitochondria and mitophagy: the yin and yang of cell death control. Circ Res 111 (9), 1208-21. 
122. Catanzaro, M. et al. (2015) Mitochondrial Fragmentation and Mitophagy Contribute to Doxorubicin-induced Cardiomyocyte Death. FASEB J 29 (1).

123. Scott, I. and Logan, D.C. (2008) Mitochondrial morphology transition is an early indicator of subsequent cell death in Arabidopsis. New Phytol 177 (1), 90-101.

124. Balk, J. and Leaver, C.J. (2001) The PET1-CMS mitochondrial mutation in sunflower is associated with premature programmed cell death and cytochrome c release. Plant Cell 13 (8), 1803-18. 125. Jones, A. (2000) Does the plant mitochondrion integrate cellular stress and regulate programmed cell death? Trends Plant Sci 5 (5), 225-30.

126. Kobayashi, H. et al. (2013) Spatial and temporal progress of programmed cell death in the developing starchy endosperm of rice. Planta 237 (5), 1393-400.

127. Lemasters, J.J. et al. (1999) Mitochondrial dysfunction in the pathogenesis of necrotic and apoptotic cell death. J Bioenerg Biomembr 31 (4), 305-19.

128. Yu, X.H. et al. (2002) Mitochondrial involvement in tracheary element programmed cell death. Cell Death Differ 9 (2), 189-98.

129. Hackenberg, T. et al. (2013) Catalase and NO CATALASE ACTIVITY1 promote autophagydependent cell death in Arabidopsis. Plant Cell 25 (11), 4616-26.

130. Munch, D. et al. (2015) Retromer contributes to immunity-associated cell death in Arabidopsis. Plant Cell 27 (2), 463-79.

131. Teh, O.K. and Hofius, D. (2014) Membrane trafficking and autophagy in pathogen-triggered cell death and immunity. J Exp Bot 65 (5), 1297-312. 


\section{Figure legends}

Figure 1. A putative model of the mechanisms of mitophagy in plants. Upon the imposition of stress, mitochondria send a signal of an unknown nature which inhibits target of rapamycin (TOR) kinase. Inhibition of TOR allows the formation of active ATG1/13 complex by dephosphorylation, together with ATG11 and ATG101, which is recruited to the surface of mitochondria. A putative receptor present on the outer or inner mitochondrial membrane (which may become exposed upon outer membrane rupture) interacts with the ATG1/13 complex and induces pre-autophagosomal structure (PAS) formation. The autophagosome is decorated with ATG8-phosphatidylethanolamine (ATG8-PE) adducts, leading to delivery and the degradation of mitochondria in the plant vacuole.

Figure 2. Regulation and role of mitophagy in plants. Conditions like natural aging and stress can lead to the induction of senescence and may be associated with mitochondrial damage. Depending on circumstances, this may lead to increased bulk autophagy or specific mitophagy. Autophagy/mitophagy may help the plant with efficient recycling of nutrients from senescent or damaged tissues, or allow tissue survival. At the end of senescence or during extreme stress conditions mitophagy may contribute to cell death. Mitophagy may also play a role during developmental cell death. Retrograde signalling can be induced by mitochondrial stress, which may contribute to prevention of cell death [108]. Images for senescent leaf and lightning were obtained freely from www.freepik.com.

\section{Box 1 Figure I. Gene expression of ATG genes during dark-induced and developmental} senescence. The transcripts of many genes encoding AuTophaGy related proteins are induced by senescence. The left data set represents dark induced senescence (columns represent number of days)[109]. The right data set represents natural developmental senescence of whole plants [110] sampled from day 19 to day 39 of growth, either 7h into the light period (AM) or 14h into the light period (PM). Some ATG genes show very rapid induction (e.g. ATG8B), while others show more gradual induction patterns (e.g. ATG7). Some ATG genes also seem to display diurnal expression patterns (e.g. AtTSPO) Colour scale indicates fold change of mRNA expression relative to the first time point of the respective data set; grey fields indicate that the gene was not represented on the CATMA microarrays. 
Table 1. Conservation of mitophagy components in plants with yeast and animals.

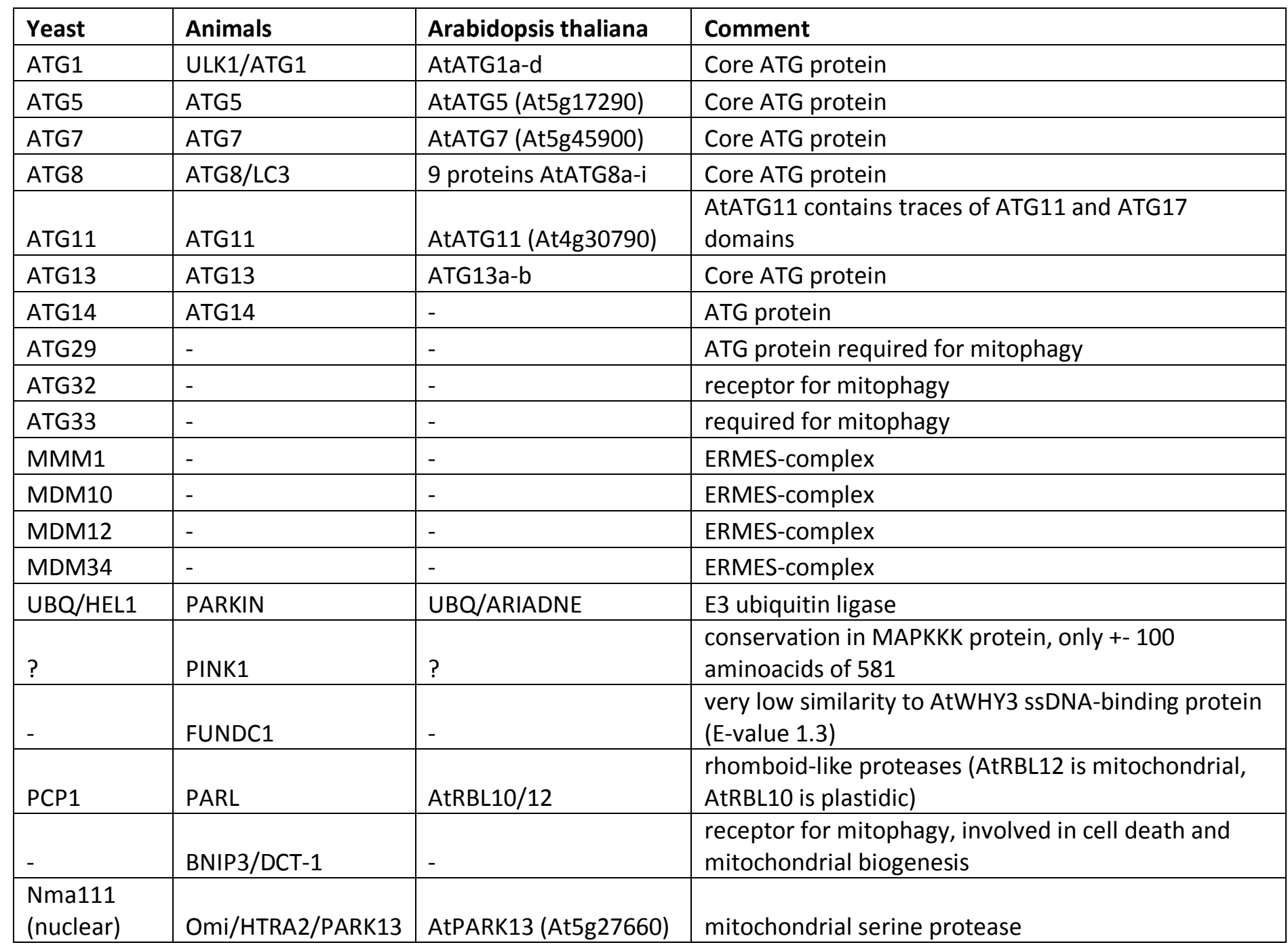


Table 2. Arabidopsis mitochondrial proteins containing an ATG8-interacting motif in. Numbers in brackets are the start position of the ATG8-interacting motif in each protein sequence. Proteins marked in bold are briefly discussed in the text.

Outer mitochondrial proteins

\begin{tabular}{|c|c|c|}
\hline AGI & Description & ATG8-interacting motif \\
\hline \multirow[t]{3}{*}{ At2g01460 } & \multirow{3}{*}{$\begin{array}{l}\text { P-Loop containing AAA+ ATPase with uridine kinase } \\
\text { domain }\end{array}$} & HDDFSSL(570) \\
\hline & & TLDFDAL(108) \\
\hline & & RNDFDPV(695) \\
\hline At5g22350 & $\begin{array}{l}\text { Elongated Mitochondria ELM1 protein of unknown } \\
\text { function (DUF1022) }\end{array}$ & HDEFAAL(248) \\
\hline At5g12290 & $\begin{array}{l}\text { DGD1 SUPPRESSOR 1, DGS1, galactoglycerolipid } \\
\text { biosyntheis }\end{array}$ & TDEWDLV(558) \\
\hline At1g05270 & TraB family protein & GEDFVHI(18) \\
\hline \multirow[t]{3}{*}{ At4g29130 } & \multirow[t]{3}{*}{ Hexokinase AtHXK1 } & DELFNFI(141) \\
\hline & & KQEFEEV(123) \\
\hline & & TLDFESL(301) \\
\hline At5g67500 & VDAC2 voltage dependant anion channel & DDIYFCL(49) \\
\hline At5g17770 & NADH:Cytochrome B5 Reductase 1 AtCBR1 & NVTYDDI(191) \\
\hline At5g20520 & Wavy-growth WAV2 prolyl oligopeptidase & NLIYEDI(51) \\
\hline At2g38280 & $\begin{array}{l}\text { Adenosine 5'-monophosphate deaminase AtAMPD } \\
\text { FAC1 }\end{array}$ & MSEWDQL(521) \\
\hline At1g27390 & $\begin{array}{l}\text { Translocase of the outer mitochondrial membrane } \\
\text { TOM20-2 }\end{array}$ & TADFERL(5) \\
\hline At3g27080 & $\begin{array}{l}\text { Translocase of the outer mitochondrial membrane } \\
\text { TOM20-3 }\end{array}$ & ETEFDRI(4) \\
\hline At3g20000 & $\begin{array}{l}\text { Translocase of the outer mitochondrial membrane } \\
\text { TOM40 }\end{array}$ & PVPYEEL(31) \\
\hline
\end{tabular}

Inner mitochondrial proteins

\begin{tabular}{|c|c|c|}
\hline AGI & Description & ATG8-interacting motif \\
\hline AT1G07180 & $\begin{array}{l}\text { Internal alternative NAD(P)H-ubiquinone } \\
\text { oxidoreductase A1; NDA1 }\end{array}$ & IDEWMRV (365-371) \\
\hline \multirow[t]{2}{*}{ AT2G20800 } & \multirow{2}{*}{$\begin{array}{l}\text { External alternative NAD(P)H-ubiquinone } \\
\text { oxidoreductase B4; NDB4 }\end{array}$} & TDEWLRV (359-365) \\
\hline & & DMDYDIL (164-170) \\
\hline AT2G29990 & $\begin{array}{l}\text { Internal alternative NAD(P)H-ubiquinone } \\
\text { oxidoreductase A2; NDA2 }\end{array}$ & IDEWMRV (363-369) \\
\hline \multirow[t]{2}{*}{ AT2G43400 } & \multirow{2}{*}{$\begin{array}{l}\text { Electron transfer flavoprotein-ubiquinone } \\
\text { oxidoreductase; ETFQO }\end{array}$} & YEEFQKL (364-370) \\
\hline & & SIEYDVL (97-103) \\
\hline \multirow[t]{3}{*}{ AT4G05020 } & \multirow{3}{*}{$\begin{array}{l}\text { External alternative NAD(P)H-ubiquinone } \\
\text { oxidoreductase B2; NDB2 }\end{array}$} & TDEWLRV (354-360) \\
\hline & & DYDYLVI (162-168) \\
\hline & & SVDYDYL (160-166) \\
\hline AT5G52840 & NADH-ubiquinone oxidoreductase-related & EEDWEMI (71-77) \\
\hline AT1G17530 & $\begin{array}{l}\text { translocase of inner mitochondrial membrane 23; } \\
\text { TIM23-1 }\end{array}$ & DDVWTSV (135-141) \\
\hline AT1G20350 & $\begin{array}{l}\text { translocase of inner mitochondrial membrane 17-1; } \\
\text { TIM17-1 }\end{array}$ & EDPWNSI (87-93) \\
\hline AT1G72750 & $\begin{array}{l}\text { translocase of inner mitochondrial membrane 23-2; } \\
\text { TIM23-2 }\end{array}$ & DDVWTSV (136-142) \\
\hline AT2G26140 & ATP-dependent zinc metalloprotease FTSH 4 & EETFGGL (138-144) \\
\hline
\end{tabular}




\begin{tabular}{|c|c|c|}
\hline & & EEMFVGV (297-303) \\
\hline AT2G37410 & $\begin{array}{l}\text { Translocase of inner mitochondrial membrane 17-2; } \\
\text { TIM17-2 }\end{array}$ & EDPWNSI (87-93) \\
\hline AT3G08580 & Mitochondrial ADP/ATP carrier; AAC1 & DEGFGSL (137-143) \\
\hline AT5G11690 & $\begin{array}{l}\text { Translocase of inner mitochondrial membrane 17-3; } \\
\text { TIM17-3 }\end{array}$ & EDPWNSI (87-93) \\
\hline AT5G25450 & Cytochrome bd ubiquinol to cytochrome c oxidase & DDL YDPL (36-42) \\
\hline \multirow{3}{*}{ AT5G53170 } & \multirow[t]{3}{*}{ ATP-dependent zinc metalloprotease FTSH 11} & EEMFVGV (432-438) \\
\hline & & VMEWEWL (158-164) \\
\hline & & LLEYETL (769-775) \\
\hline AT1G14560 & CoA transporter & FYIYEEL (209-215) \\
\hline AT2G07698 & ATPase F1 complex, alpha subunit protein & LIIYDDL (538-544) \\
\hline AT2G47690 & NADH-ubiquinone oxidoreductase-related & RTIFDEV (12-18) \\
\hline AT3G52300 & ATP synthase D chain, mitochondrial; ATPQ & RRAFDEV (41-47) \\
\hline AT4G02580 & NADH-ubiquinone oxidoreductase & YNYFEDV (195-201) \\
\hline AT5G56450 & metabolite transporter, substrate carrier & LVFYDEV (315-321) \\
\hline AT5G66380 & folate transporter 1; FOLT1 & FTAYEEL (183-189) \\
\hline ATMG01190 & ATP synthase subunit 1 & LIIYDDL (268-274) \\
\hline \multirow[t]{2}{*}{ AT5G08740 } & \multirow[t]{2}{*}{ Alternative $\mathrm{NAD}(\mathrm{P}) \mathrm{H}$-ubiquinone oxidoreductase $\mathrm{C} 1$} & EYDWLVL (192-198) \\
\hline & & KIEYDWL (190196) \\
\hline AT5G66510 & Gamma carbonic anhydrase 3; GAMMA CA3 & DTEYDSV (249-255) \\
\hline AT1G19580 & Gamma carbonic anhydrase 1; GAMMA CA1 & VIEFEKV (224-230) \\
\hline AT2G02050 & NADH-ubiquinone oxidoreductase B18 subunit & KCEYELV (60-66) \\
\hline AT2G33040 & $\begin{array}{l}\text { Gamma subunit of mitochondrial ATP synthase; } \\
\text { ATP3 }\end{array}$ & NVEFDAL (190-196) \\
\hline AT5G08530 & $\begin{array}{l}\text { NADH Coenzyme Q oxidoreductase; complex } 1 \\
\text { subunit; CI51; NDUFV1 }\end{array}$ & LMDFDAL (359-365) \\
\hline \multirow[t]{2}{*}{ AT4G21490 } & \multirow{2}{*}{$\begin{array}{l}\text { External alternative NAD(P)H-ubiquinone } \\
\text { oxidoreductase B3; NDB3 }\end{array}$} & TDEWLRV (352-358) \\
\hline & & DVDYDYL (158-164) \\
\hline AT1G09575 & Calcium uniporter protein 1; MCU1 & KEEFNKL (148-154) \\
\hline AT4G16700 & Phosphatidylserine decarboxylase proenzyme 1; PSD1 & LEEYTSL (166-172) \\
\hline AT1G47420 & Succinate dehydrogenase subunit 5; SDH5 & VEEFGGI (154-160) \\
\hline AT3G59280 & $\begin{array}{l}\text { Mitochondrial import inner membrane translocase } \\
\text { subunit PAM16 like } 2 \text { (AtPAM16) }\end{array}$ & KTSWEEI (67-73) \\
\hline AT5G66650 & Calcium uniporter protein MCU6 & RQEFEQL (198-204) \\
\hline AT5G58270 & ABC transporter B family member 25; ABC25 & NIEFENV (478-484) \\
\hline
\end{tabular}




\section{Box 1: Control and initiation of mitophagy in plants}

As mitochondria are a significant source of ROS in plants, they are likely to be targets of autophagy in stress conditions [12]. Autophagy is thought to be induced by the plant hormone salicylic acid via NPR1 to act as a negative feedback loop repressing senescence and programmed cell death [18, 71, 111]. Oxidative stress triggered by ETC inhibitors such as antimycin A (AA) or methyl viologen (MV) was found to induce high levels of plant autophagy [2]. This effect could be overcome by exogenous addition of antioxidants. A more detailed investigation of the impact of the ETC inhibitors myxothiazol, AA or potassium cyanide (KCN) on yeasts has confirmed that AA and KCN can induce autophagy [112] as reported by Minibayeva and colleagues in plants [2]. However, Deffieu and co-workers [112] claimed that AA and KCN induced non-specific autophagy rather than mitophagy, whereas myxothiazol induced autophagy to a lesser extent. Like AA, myxothiazol blocks complex III, but it is thought to result in far less superoxide formation than AA [113]. These results suggest that autophagy is a response to ROS formation itself, rather than energy organelle inhibition. Also conditions such as hypoxia, mitochondrial uncoupling and loss of $\Delta \psi_{\mathrm{m}}$ are all known triggers for mitophagy in animal systems [114] , but have not been studied extensively in plants.

Posttranslational modification of proteins such as ubiquinylation, phosphorylation and acetylation are important in the regulation of mitophagy levels in the eukaryotic cell [115, 116]. It is already known that dephosphorylation of ATG1 and ATG13 plays crucial role in the nutrient starvation-induced activation of the ATG1/13 complex, which is required for autophagosome formation, in yeast [117], and potentially in plants [57]. In animals, the phosphoregulation of the ATG1/ATG13 complex appears to be more complex $[118,119]$ (Figure 1) .

Two starvation conditions are widely used as triggers in autophagy studies in plants: nitrogen starvation and carbon starvation [19, 120]. Nitrogen starvation seems to be a trigger for the induction of mitophagy in plants [19] and yeast [112]. The carbon status and sugar levels may 
also play a role in plant autophagy. Environmental changes like the intensity of light, access to water and temperature influence the level of carbohydrate supply. Aubert et al. suggest that the supply of mitochondria with respiratory substrates, and not the decrease of sucrose and hexose phosphates, controls the induction of bulk autophagy in plant cells starved in carbohydrates [120]. Altogether, nutrient homeostasis of the cell and the respiratory status of mitochondria are linked, and both are likely to be important in deciding between bulk autophagy and selective autophagic processes like mitophagy. 


\section{Box 2: Role of mitophagy in deciding between survival and death}

Mitophagy has been studied as a mechanism to improve cell survival by removal of damaged component or recycling nutrients [121], but excessive levels of autophagy could tip the balance towards cell death [5, 122](Figure 2). A key mechanism that affects autophagy appears to be mitochondrial fragmentation. A highly fragmented mitochondrial pool is more easily degraded by mitophagy, while a highly aggregated mitochondrial pool may be more resistant [122]. The fact that plant mitochondria aggregate early during cell death [123] may contribute to a failing of mitophagy to rescue the cell.

The role of mitochondria in PCD has been studied for some time [124, 125] but the role of autophagy in plant PCD is not very well understood, with a few notable exceptions. A recent study demonstrated that during developmental PCD of suspensor cells in Norway Spruce a metacaspase- and autophagy dependent pathway is used, but in their absence a mitochondrial PCD pathway was observed [17]. In rice starchy endosperm PCD, mitochondrial membrane permeabilisation and caspase-like activity preceded cell death, suggesting mitochondrial PCD and autophagy are not necessarily mutually exclusive during plant developmental PCD [126]. Some studies have suggested that mitochondria undergoing permeability transition (MPT) become targeted for autophagy, so widespread MPT inside a cell following pro-death signals may trigger cell death by excessive removal of mitochondria by autophagy [127]. Arabidopsis mutants in the mitochondrial protease Ftsh4 displayed increased senescence, PCD and autophagy. Crossing with atg5 or atg8 mutants reduced PCD levels and reversed early leaf senescence, suggesting that autophagy stimulated both leaf senescence and PCD in this protease mutant [71]. In agreement, many of the Arabidopsis ATG genes are transcriptionally regulated during leaf senescence [109] (Box 1 Figure I). Wertmann and colleagues described macro- and mega autophagy during lace plant PCD [27]. Many autophagic vesicles were being formed during early PCD stages. These vesicles contained organelle aggregates which often 
co-stained with mitochondria already during early stages of PCD stages. These aggregates migrated to the vacuole in late stage PCD, suggesting mitophagy is part of the PCD process. Autophagy is necessary for PCD in developmental tracheary element formation in the xylem [26] and mitochondria have a role in triggering PCD during tracheary element formation [128]. Mitophagy has been observed during the first day of tracheary element induction with brassinolide $/ \mathrm{H}_{3} \mathrm{BO}_{3}$, while a brassinosteroid-insensitive mutant did not show this process, indicating the involvement of phytohormones [26]. Finally, autophagy may also play a role in plant immunity and pathogen-induced PCD, a process potentially downstream of catalase function, linking ROS production with autophagy-dependent PCD [129-131]. In summary, it seems that in plants autophagy may be both a suppressor and stimulator of PCD processes. 


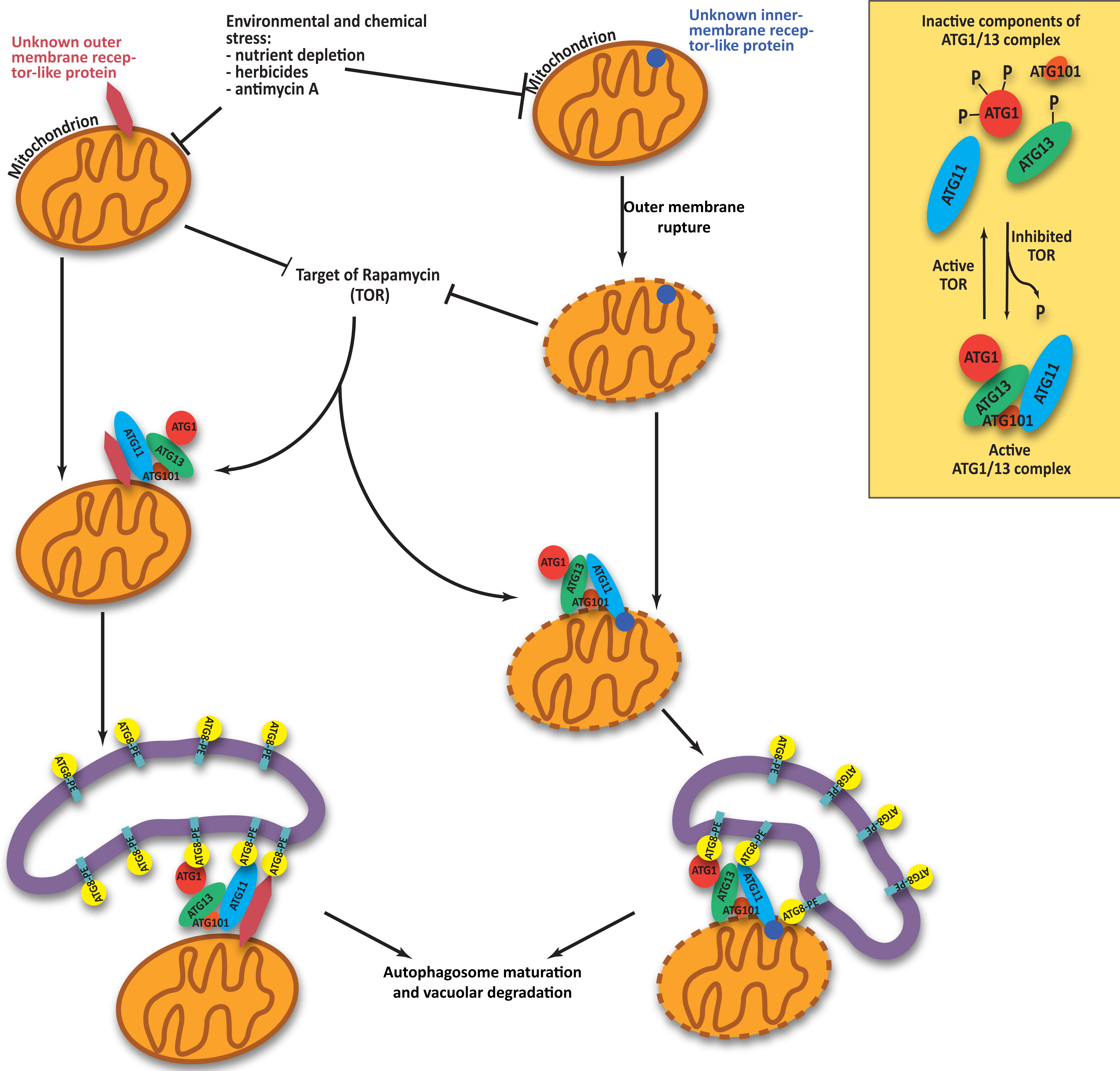




\section{Natural ageing}

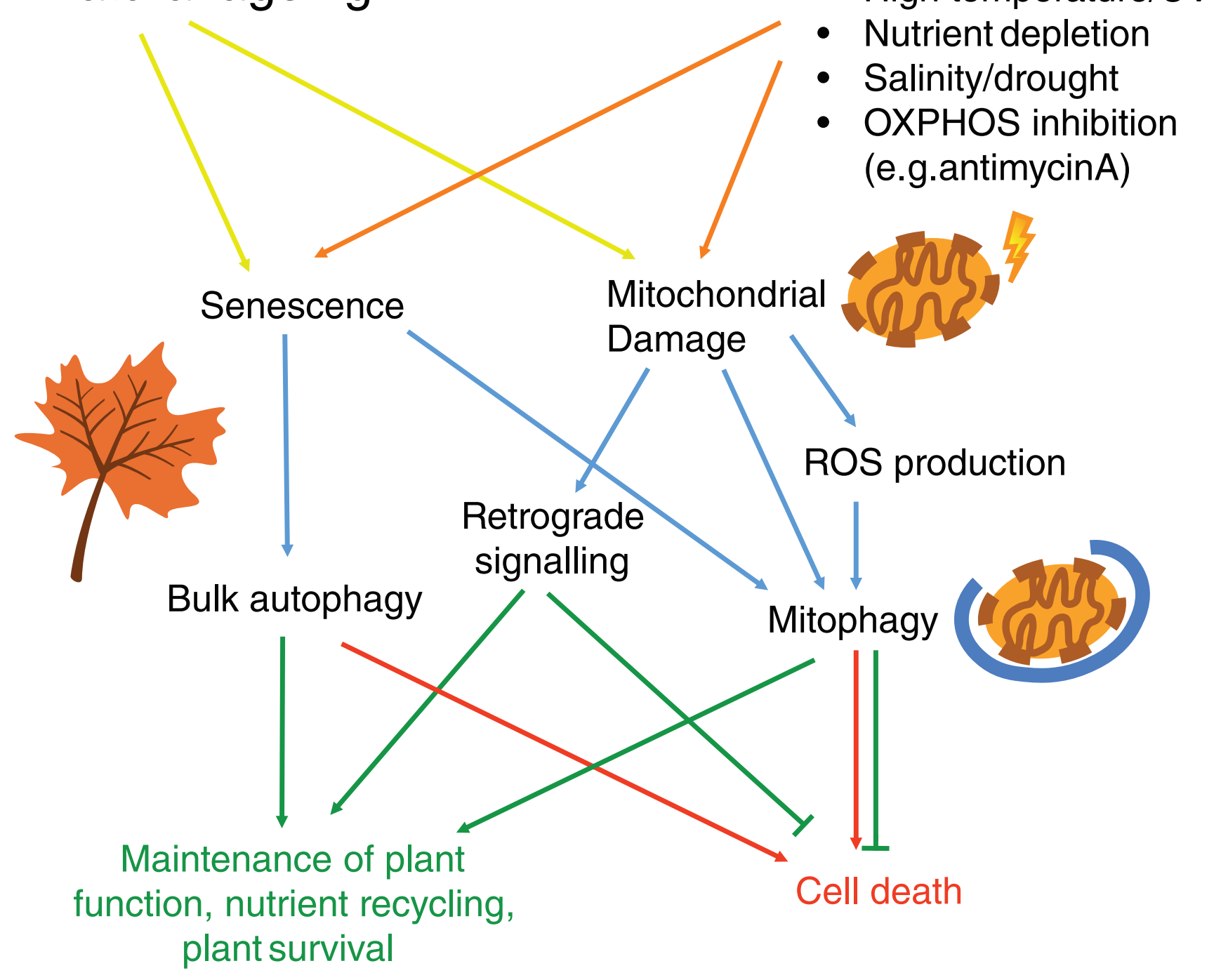




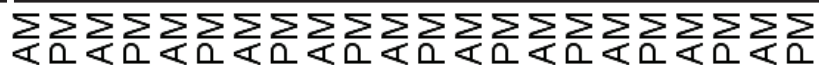

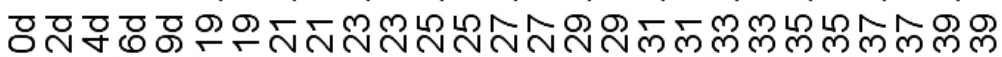

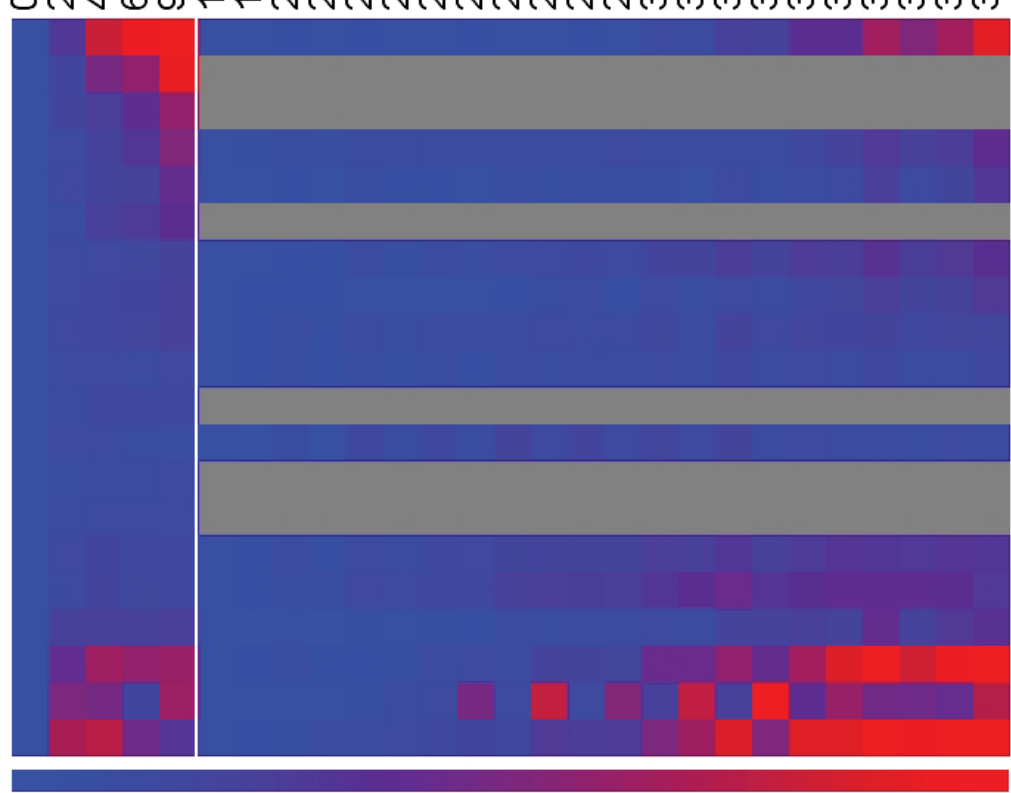

ATG7 AT5G45900

ATG8̄ AT3G06420

ATG8E-AT2G45170

ATG9 ĀT2G31260

ATG11_AT4G30790

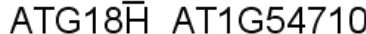

ATG101-AT5G66930

ATG18A AT3G62770

NBR1 AT4G24690

ATG5 ${ }^{-A T 5 G 17290}$

ATG3-AT5G61500

ATG8̄ AT4G16520

ATG8D AT2G05630

ATG8C-AT1G62040

ATG8I AT3G15580

ATG8G AT3G60640

ATG13-AT3G49590

ATG8A- AT4G21980

AtTSPO AT2G47770

ATG8B_AT4G04620 